\title{
Competencia para el cuidado (CUIDAR) en el hogar de personas con enfermedad renal crónica en hemodiálisis
}

\author{
Erika Alexandra Aldana, Sandra Yomaira Barrera, Konniev Alexei Rodríguez, Olga Janeth Gómez, Gloria Mabel \\ Carrillo
}

Dialy-ser IPS Renal. Universidad Nacional de Colombia Facultad de Enfermería. Bogotá. Colombia

\section{Resumen}

Objetivo: Describir la competencia para el cuidado en el hogar (CUIDAR) de personas con enfermedad crónica que se encuentran en hemodiálisis.

Materiales y método: Estudio cuantitativo, descriptivo, prospectivo y de corte transversal, desarrollado entre el 2014 y 2015. La muestra estuvo conformada por 131 pacientes con pacientes en hemodiálisis que asisten a un centro de atención en terapia renal de la ciudad de Bogotá. Se emplearon los instrumentos "Encuesta de caracterización para el cuidado de una persona con enfermedad crónica" y "Competencia para cuidar en el hogar GCPC-UN-CPC, versión paciente". Se utilizó estadística descriptiva para analizar la competencia para cuidar en el hogar y sus dimensiones a partir de los niveles de estratificación alto medio y bajo.

Resultados: Las personas con enfermedad renal crónica en hemodiálisis presentan niveles bajos y medios de competencia para cuidar en el hogar. Las dimensiones unicidad y disfrutar reportan los niveles más bajos, en contraste, el componente de relación e interacción social está dentro de los niveles más altos.

Conclusiones: La competencia para cuidar en el hogar de personas en hemodiálisis en el hogar se en-

Correspondencia:

Gloria Mabel Carrillo González

Universidad Nacional de Colombia. Facultad de Enfermería Calle $44 N^{\circ}$ 45-67 Bloque B2, Oficina 801

Carrera 48 N. 22-80 Manzana B, casa 44

Bogotá. COLOMBIA

E-mail: gmcarrillog@unal.edu.co cuentra en niveles desiguales que pueden afectar la continuidad y seguridad, es necesario fortalecer dicha competencia en cada uno de sus componentes como beneficio concreto en la promoción de la salud en este grupo de personas.

Palabras clave:

- INSUFICIENCIA RENAL CRÓNICA

- DIÁLISIS RENAL

- ALTA DEL PACIENTE

- SEGURIDAD DEL PACIENTE (DESCRIPTORES DECS)

Competition for the care (CARE) at the home of people with chronic kidney disease on hemodialysis

\section{Abstract}

Objective: To describe the competition for home care (CARE) for persons with chronic disease on hemodialysis.

Materials and Methods: Quantitative, descriptive, prospective and cross-sectional study conducted between 2014 and 2015. The sample consisted of 131 hemodialysis patients attending to a renal therapy center in Bogotá city. The instruments used were: "Characterization Survey to Define the Care of a Person with Chronic Disease" and "Competition for care at home GCPF-UN-CPC, patient version". Descriptive statistics was used to analyze the competition for care at home and their dimensions from stratification levels (high, medium and low). 
Results: People with chronic kidney disease on hemodialysis have low and medium levels of competence to care at home. The dimensions of "uniqueness" and "enjoy" report the lowest levels, in contrast, the components of relationship and social interaction are among the highest levels.

Conclusions: The competence for care at home for people in home hemodialysis is in unequal levels which can affect the continuity and security. That competence must be strengthened, in each of its components as a specific benefit in promoting health in this group of people.

\section{KEYWORDS}

- CHRONIC KIDNEY DISEASE

- RENAL DIALYSIS

- PATIENT DISCHARGE

- PATIENT SAFETY

\section{Introducción}

La insuficiencia renal generada cuando la tasa de filtración glomerular es menor de $15 \mathrm{ml} / \mathrm{min} / 1,72 \mathrm{~m}^{2}$ y la enfermedad renal crónica (ERC) avanzada, son entidades que implican la posibilidad de terapia de sustitución o reemplazo (diálisis o trasplante), tiene una alta mortalidad en países en desarrollo, en parte por el aumento en el riesgo cardiovascular. Esta mortalidad puede verse afectada por diversos factores entre los que se destacan los socioeconómicos, las comorbilidades preexistentes, las mismas terapias sustitutivas en sí, y la complejidad de la enfermedad renal. Así mismo situaciones como el acceso oportuno a la terapia de reemplazo renal y la optimización de la atención clínica puede impactar en la disminución de la mortalidad ${ }^{1,2}$.

Para Colombia la prevalencia de ERC es de 658 pacientes por millón de habitantes según datos publicados por la Cuenta de Alto Costo del Fondo Colombiano de Enfermedades de Alto Costo en el 2013³. En Colombia, 2.579.739 pacientes tienen alguna enfermedad precursora para ERC y de estos, 975.479 tienen diagnosticado algún estadio de ERC dentro de la población afiliada a las aseguradoras que hacen parte del Sistema General de Seguridad Social en Salud.

En relación con la terapia de reemplazo renal, cifras del 2013 en Colombia ${ }^{3}$ reportan que la mayor proporción de los pacientes con ERC estadio 5 (23.995) se encuentran en hemodiálisis (HD) $(55.5 \%)$ hallazgos que coinciden con la situación mundial ${ }^{4,5}$.

La HD es un procedimiento invasivo que se constituye en un reto para los profesionales encargados del cuidado porque conlleva alto riesgo de complicaciones inherentes a la terapia en sí misma y compromete la calidad de vida de los pacientes ${ }^{6}$; trae consigo cuestionamientos sobre la adherencia terapéutica de los enfermos respecto a los medicamentos que se deben tomar, el seguimiento de la dieta o los cambios en los estilos de vida ${ }^{7}$.

En el marco del modelo para abordar el cuidado de la enfermedad crónica en Colombia ${ }^{8}$ se identifica que la competencia para el cuidado en el hogar es un aspecto fundamental dentro del quehacer del equipo de salud facilitando que los pacientes logren llegar a su hogar con la seguridad de saber cómo dar continuidad al manejo de su condición de salud. Estudiar la competencia para el cuidado es particularmente necesario para la promoción de la calidad de vida de los pacientes con ERC en $\mathrm{HD}$, teniendo en cuenta los múltiples aspectos para su cuidado, tales como la necesidad de educación, de acompañamiento y seguimiento para poder cuidar mejor su salud y fortalecer su propia capacidad de cuidado.

Para las instituciones de salud, abordar la competencia de cuidado en el hogar se constituye en una estrategia de intervención con la que se garantiza el cuidado seguro del paciente, el apoyo al cuidador familiar y la respuesta a parámetros de calidad que evidencien que el cuidado que se brinde sea exitoso dentro y fuera del ámbito hospitalario.

En tal sentido, el objetivo del presente estudio fue determinar cuál es la competencia para el cuidado en el hogar (CUIDAR) de los usuarios con ERC que reciben terapia renal de HD y asisten a la IPS Dialyser en la ciudad de Bogotá-Colombia.

\section{Metodología}

Estudio de abordaje cuantitativo, prospectivo de tipo descriptivo, y de corte transversal, realizado entre el 2014 y 2015. La muestra estuvo conformada por 131 personas con enfermedad renal crónica que asisten a HD en la IPS Dialyser Bogotá Colombia.

El estudio contó con los respectivos avales institucionales de la IPS Dialyser y éticos de la Universidad Nacional de Colombia en el marco del Programa para 
Disminuir la carga de la enfermedad crónica, se aplicó el procedimiento de consentimiento informado a los pacientes quienes voluntariamente aceptaron participar del estudio y se aplicaron todos los requisitos exigidos por la norma 8430 de 1993 para la investigación en salud en Colombia9. Sus autores se comprometieron con la política de protección del medio ambiente y en especial con el uso racional de recursos ${ }^{10}$.

\section{Variables:}

1. Perfil y datos sociodemográficos del paciente: género, edad, tiempo que lleva con la enfermedad, escolaridad, estado civil, diagnóstico médico. presencia de un cuidador, apoyos con los que cuenta; percepción de bienestar físico, psicoemocional, social y espiritual; y tecnologías de información y la comunicación para el cuidado.

2. Competencia para el cuidado en el hogar: Es la capacidad, habilidad y preparación que tiene la persona con enfermedad crónica para ejercer la labor de cuidar en el hogar. Incluye 6 categorías: Conocimiento, Unicidad (condiciones personales), Instrumental, Disfrutar (bienestar), Anticipación, y Relación social e interacción.

\section{Instrumentos:}

Encuesta de caracterización para el cuidado de una "persona con enfermedad crónica".

Contiene 22 ítems y tres dimensiones: a) Las condiciones y perfil socio demográfico de la Diada (persona con IRC-Cuidador familiar). b) La percepción de carga y apoyo. c) Los medios de información y comunicación.

La encuesta desarrollada y validada cuenta con las variables necesarias para la caracterización de una persona con enfermedad crónica en el contexto de América Latina $^{11}$.

Competencia para el cuidado en el hogar CUIDAR (GCPC-UN-CPC) paciente con enfermedad crónica versión abreviada. Está conformada por 20 ítems que se miden con una escala tipo Likert con puntuaciones que van de 0 a 3; 0 es nunca o casi nunca, 1 pocas veces, 2 con frecuencia y 3 casi siempre o siempre. Cuenta con las pruebas psicométricas para ser usado en Colombia incluidas validez aparente, validez de constructo y confiabilidad. Para esta aplicación se obtuvo una consistencia interna dada por un alfa de Cronbach de $0.91^{12}$.

Marco de análisis: Los hallazgos reportados a través de los instrumentos fueron trasladados a una base de datos
Excel, y analizados en el programa SPSS versión 22.0, bajo licencia de la Universidad Nacional de Colombia.

Para la caracterización de las personas con enfermedad crónica, se utilizó estadística descriptiva con presentación de frecuencias absolutas y relativas. Respecto a la competencia para el cuidado en el hogar de personas con enfermedad crónica, se aplicó estadística descriptiva utilizando medidas de tendencia central y de dispersión para la totalidad del instrumento y las dimensiones. Adicionalmente, la competencia para el cuidado en el hogar se analizó a partir de los niveles de estratificación propuestos: alto medio y bajo.

\section{Resultados}

Caracterización de las personas con enfermedad renal crónica en HD

En el 59,5\% de los participantes hay dependencia leve, un importante grupo reporta niveles de dependencia moderada $(26 \%)$ a severa $(13,7 \%)$, relacionada muy probablemente con las secuelas de la ERC y con las complicaciones propias del tratamiento dialítico.

Diagnósticos médicos: Los hallazgos del estudio respecto a esta variable coinciden con la presencia de enfermedad renal crónica con comorbilidades asociadas tales como diabetes, hipertensión arterial y retinopatías especialmente. (Tabla 1).

Tabla 1. Tipo de enfermedades crónicas de los participantes en Dialyser IPS.

\begin{tabular}{|lr|}
\hline Enfermedad & Porcentaje \\
\hline Diabetes & $35,37 \%$ \\
\hline Hipertensión & $52,44 \%$ \\
\hline Glomerulonefritis & $4,88 \%$ \\
\hline Lupus Sistémico & $2,85 \%$ \\
\hline Obstructiva & $2,85 \%$ \\
\hline Enf Poliquística & $1,63 \%$ \\
\hline Total & $\mathbf{1 0 0 , 0 0 \%}$ \\
\hline
\end{tabular}

Fuente: Datos de la investigación, 2015.

Dentro de los participantes hay una mayoría masculina (61,8\%). En cuanto a la edad la mediana es de 60 años y un promedio de 57,7 años; el $12,2 \%$ de los usuarios tiene 35 años o menos, el $37,4 \%$ de 36 a 59 años, el $39,7 \%$ de 60 o 75 años y el 9,9\% entre 76 y 80 años. 
Respecto al nivel educativo, el 7,6\% de los pacientes es analfabeta, el $42,7 \%$ tiene primaria, el $13 \%$ bachillerato incompleto, el $25,2 \%$ bachillerato completo, nivel técnico el 7,7\% y únicamente el 3,8\% refiere contar con nivel universitario completo. El $92,4 \%$ de las personas proceden de la zona urbana. El $66.4 \%$ de los participantes indica contar con una unión de pareja estable.

Frente a la ocupación el $51,1 \%$ refiere no tener una actividad económica, el $11,5 \%$ es empleado, el $16,8 \%$ cuenta con algún trabajo independiente, el $15,3 \%$ está pensionado, el 3,8\% es estudiante y el 1,5\% manifiesta no tener ninguna ocupación. La mayor parte de los participantes pertenecen a estrato socioeconómicos bajos (1: 16\%; 2: 52,7\%).

En cuanto al tiempo que lleva con la enfermedad el $71,8 \%$ tiene 5 años o menos, el 3,1\% entre 6 a 10 años, el $8,4 \%$ entre 11 y 15 años y el 5,3\% más de 16 años.

El $83,9 \%$ de las personas reconoce tener un cuidador familiar a cargo. El 58,8\% Refiere requerir el apoyo de un cuidador familiar seis horas o menos al día, en contraste con el $13 \%$ que lo requiere durante 24 horas. En el $48,9 \%$ de los casos la persona a cargo del cuidado es el esposo/a del paciente, en el $19,1 \%$ los hijos, seguidos por el $10,7 \%$ en el que son los padres.

Con respecto a los apoyos percibidos están el psicológico en el 75,6\%, sin embargo, el $64,9 \%$ no están satisfechos con el apoyo que reciben. En cuanto al apoyo familiar el $98,5 \%$ señalan percibirlo, solo $10,7 \%$ no se encuentran satisfechos con este.

Frente al apoyo social el $46,6 \%$ de los participantes lo percibe y expresa satisfacción con el mismo. El $70,2 \%$ refiere contar con algún tipo de apoyo económico. El $27,5 \%$ de las personas siente que es una carga alta o muy alta para su familia.

Los participantes perciben bienestar físico en el $52,7 \%$ de los casos, psicológico en el $71,8 \%$, social en el $67,9 \%$ y espiritual en el $83,2 \%$.

La valoración del nivel de apropiación de las tecnologías de información y comunicación (TIC) refleja alto conocimiento, acceso y uso de la televisión, seguido por la radio y el teléfono. En un porcentaje muy bajo los usuarios reconocen el ordenador e internet como elemento fundamental para facilitar o apoyar el cuidado de la salud en las situaciones de EC. EI 57,3\% de las personas no usa TIC para apoyar su cuidado (Figura 1).

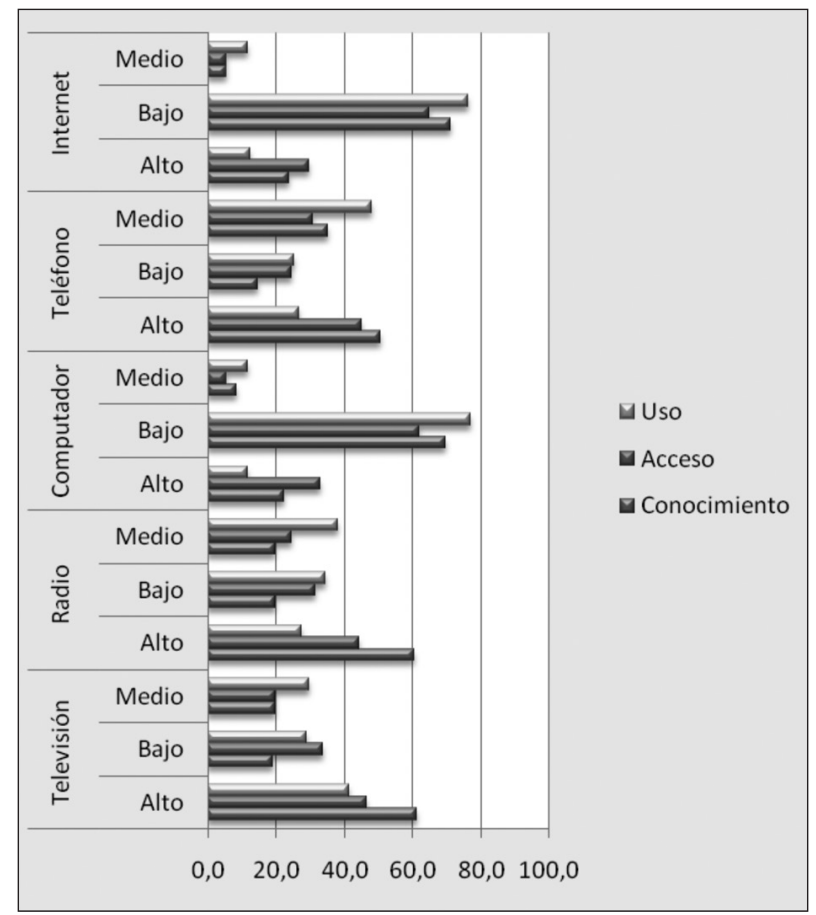

Figura 1. Apropiación en porcentaje (\%) de las tecnologías de información y comunicación en personas con enfermedad renal crónica en terapia renal.

Fuente: Datos de la investigación, 2015.

\section{Competencia para el cuidado en el hogar CUIDAR}

La competencia para el cuidado en el hogar de personas con enfermedad renal crónica participantes del estudio indica con puntuaciones posibles de 0 a 60 una media de 45,5 y una desviación estándar (DS) de 8,4. En las dimensiones, conocimiento (puntuación posible 0-9) presentó un promedio de 6,25 DS 1,9; unicidad (puntuación posible 0-12) 8,17 DS 2,1; instrumental (puntuación posible 0-9) 6,85 DS 1,5; disfrutar (puntuación posible 0 a 12) 6,76 DS 2,5; anticipación (puntuación posible 0 a 6) 4,13 DS 1,2 y relación e interacción (puntuación posible 0 a 12) de 10,37 DS 2,3 (Figura 2). 


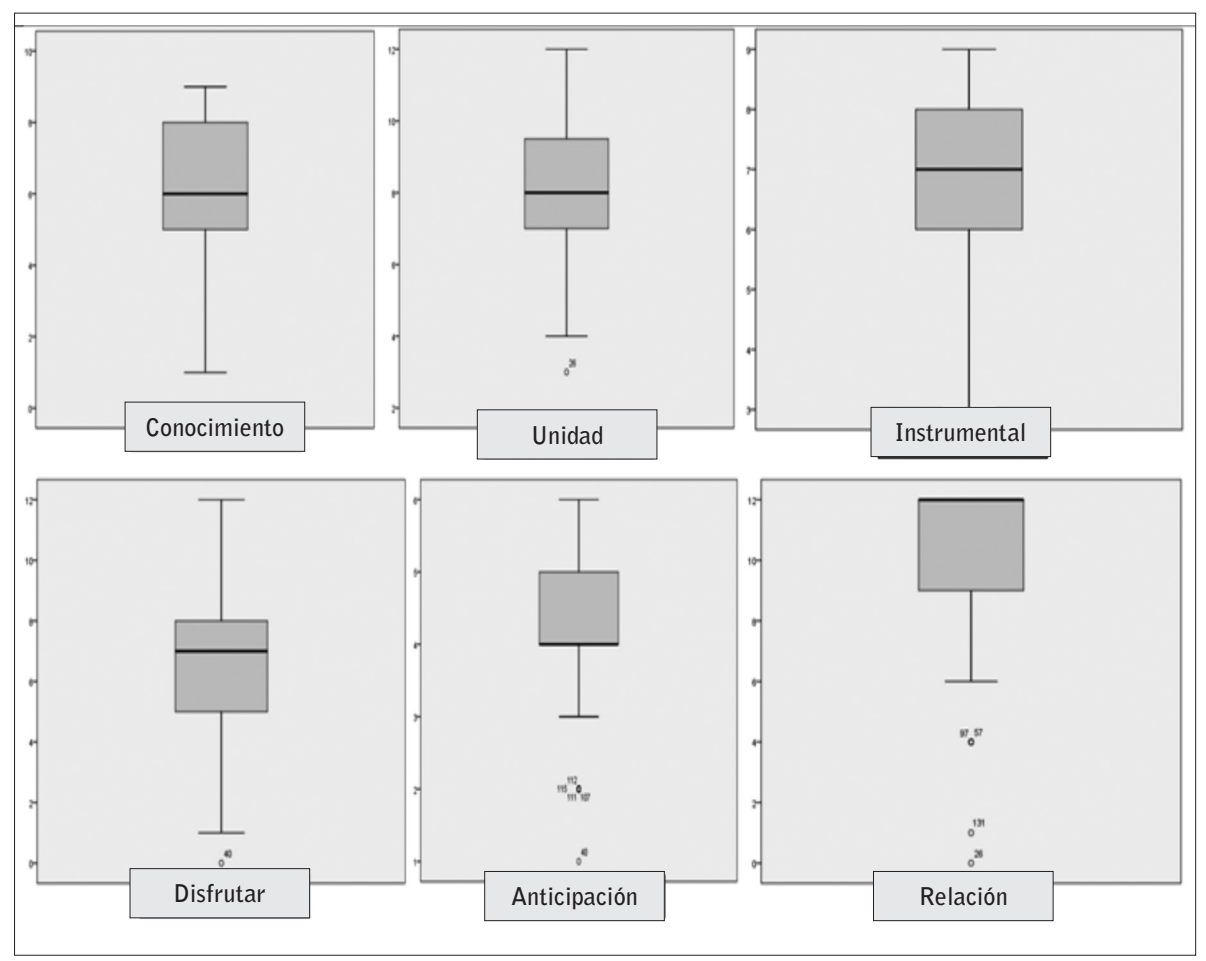

Figura 2. Competencia para el cuidado en el hogar (CUIDAR) personas con enfermedad renal crónica en hemodiálisis.

Fuente: Datos de la investigación, 2015.

Por niveles, se identificó que solo el $28,8 \%$ de las personas con ERC presentan un alto nivel de competencia para el cuidado en el hogar, el $44,7 \%$ reporta un nivel medio y el $25,8 \%$ bajo.

Respecto a las dimensiones se identifica que los valores altos obtenidos están en Conocimiento del $40,2 \%$, en Unicidad sólo el $25 \%$, en Instrumental el $34,8 \%$, en

\section{Discusión}

Disfrutar el 23,5\%, en Anticipación el $28 \%$ y en Relación el $65,2 \%$. (Figura 3 ).

Al buscar identificar si existe relación entre las variables sociodemográficas de los participantes y su competencia para el cuidado en el hogar, sólo se identificó asociación estadísticamente significativa $(p<0,005)$ débil entre la edad en años de los participantes (coeficiente Spearman -0,27) y el número de horas de ayuda que requiere diariamente para su cuidado (coeficiente Spearman -0.23). Se identifica además que hay diferencias estadísticamente significativas $(p<0,005)$ a través de la prueba de $U$. de Mann Whitney en la competencia para el cuidado en el hogar (CUIDAR) entre hombres $y$ mujeres, siendo mayor en el género femenino particularmente en las dimensiones conocimiento e instrumental.

En esta investigación la ERC se constituye como una problemática presente en todos los grupos de la misma edad, afectando de manera especial a los adultos mayores con una mediana de 60 años, hallazgos que se relacionan con lo reportado a nivel nacional en el informe de la cuenta de alto costo 2013 que muestran una proporción mayor de HD en personas mayores de 55 años, Sanabria et al en 2008 reportaron una mediana de 57 años para pacientes en $\mathrm{HD}^{13}$, sin embargo es menor a los referido por Coronado et $\mathrm{a}^{14}$, quienes identificaron para su estudio con pacientes que iniciaban terapias renales una mediana de 65 años.

Se identifica además que un grupo de usuarios $(39,7 \%)$ dimensiones: clasificación por niveles.

Fuente: Datos de la investigación, 2015. 
evidencia niveles de dependencia moderada y alta, aspecto que pone significativas exigencias a los sistemas de salud y bienestar social, al afectar probablemente la productividad y empobrecer a las familias ${ }^{15}$. Esta situación se relaciona estrechamente con los resultados respecto a la ocupación hogar como la más alta, siendo llamativo el bajo porcentaje de personas que se encuentran empleados.

Por otro lado, desde el componente sociocultural se identifica una tendencia a la estigmatización del enfermo renal como paciente dependiente, con alta necesidad de un cuidador familiar, aspectos que aunque no fueron explorados en este estudio, deben ser sujeto de análisis ya que esta variable, es determinante para optar por pensión asociada a invalidez y alta dependencia.

Respecto a la escolaridad es particularmente los bajos niveles educativos de los usuarios del estudio, siendo marcada la población analfabeta. La educación ha sido asociada con la prevención de las Enfermedad Crónica (EC) señalando una relación positiva entre el nivel educativo y la conducta preventiva ${ }^{16,17}$, más aún, hay reportes de mayor prevalencia de EC entre hombres y mujeres viejos cuando estos tienen menor nivel de escolaridad ${ }^{18}$. Se constituye en un desafío para el talento humano en salud, en el marco de la generación de propuestas de intervención para los enfermos renales crónicos orientadas a la educación y capacitación.

En cuanto al estado civil, se reconoce que un alto porcentaje de enfermos renales crónicos cuentan con una relación estable por matrimonio o vínculo de unión libre. El hecho de contar con una pareja es un factor de ayuda para muchos pacientes, cuando se deben tomar decisiones difíciles. En la relación entre esposos, cuando uno de los miembros de la pareja sufre un deterioro de la salud y necesita ayuda para sus actividades de la vida diaria, el cuidador principal suele ser el de mejor salud, pero en el proceso puede terminar deteriorándose ${ }^{19}$.

Los hallazgos del estudio evidencian que las personas con ERC que asisten a la IPS Dialyser son en su mayoría de estratos socioeconómicos bajos y medios, situación que se relaciona estrechamente con la zona de residencia de los usuarios, la ocupación y el nivel de escolaridad. Se identifica un grupo mayoritario de personas que llevan de 0 a 5 años con ERC. Las diferencias de percepciones cambian en los diferentes periodos, sin embargo, las variaciones más importan- tes se presentan en los primeros seis meses ${ }^{19}$. Ratificándose la necesidad de realizar un abordaje educativo integral por parte del equipo interdisciplinario, con especial énfasis en los primeros meses de la terapia.

Ahora bien, la satisfacción negativa con el soporte social, el tamaño de la red de apoyo, y el estado funcional son predictores significativos de soledad ${ }^{20}$. Para muchos el apoyo emocional es más importante que el apoyo instrumental. En ausencia de acontecimientos estresantes, una amplia red social puede promover la salud. En la presencia de estrés, el soporte funcional puede ser importante para mejorar las tensiones. El apoyo instrumental y emocional está alto e inversamente relacionado con la depresión y los síntomas de ansiedad. Existe un efecto amortiguador moderado entre la participación familiar y los síntomas psicológicos que se relacionan con la capacidad de los sujetos para afrontar el deterioro cognitivo y las enfermedades crónicas ${ }^{21}$.

Los pacientes que tienen un elevado nivel de satisfacción con el soporte social, por parte de la familia, los amigos y los profesionales, bien sea emocional 0 instrumental, experimentan menos efectos negativos de las crisis. Por el contrario, la soledad y la falta de apoyo, generan sufrimiento ${ }^{19}$.

En relación con la carga percibida se identifica que un porcentaje significativo de las personas con ERC perciben ser una carga alta para su familia, en este sentido se puede inferir que el manejo de la enfermedad por parte del paciente conlleva a una restricción de su vida social. A menor funcionalidad, mayor carga y sentimientos de depresión lo cual termina repercutiendo en la diada persona con enfermedad crónica y su cuidador familiar ${ }^{19}$. Se ha encontrado que la percepción de carga de la persona enferma se asocia a su bienestar espiritual22; también que el bienestar emocional predice el pronóstico a largo plazo de la enfermedad física ${ }^{23}$.

En cuanto a la competencia para el cuidado en el hogar (CUIDAR) se identifica que la mayor parte de los participantes se encuentran en rangos bajo y medio, hallazgo que es similar al reportado en un estudio de carácter nacional desarrollado con personas que presentan enfermedad crónica, siendo evidente la heterogeneidad en los niveles encontrados de CUIDAR, los cuales no llegan a ser óptimos ${ }^{24}$.

Respecto a las dimensiones, se identifica que las que reportan los niveles más bajos son disfrutar (Bienes- 
tar) y Unicidad. El componente de unicidad (condiciones personales) corresponde a las características tanto internas como de personalidad, base fundamental para que se afronte de una u otra forma la enfermedad crónica y el rol como persona enferma o cuidador. De estas condiciones se conciben todas aquellas formas y herramientas con las que se cuenta para hacer frente a problemas, retos y desafíos, así como mecanismos de afrontamiento para superarlos de la mejor manera.

En la dimensión disfrutar (bienestar) se indaga sobre el grado de bienestar que se tiene para comprender como se asume la responsabilidad del cuidado en casa en aspectos básicos de la vida cotidiana, así como la satisfacción personal relacionada con la calidad de vida percibida.

Los hallazgos del estudio se relacionan estrechamente con lo referido por Quinan ${ }^{25}$ quien señala que el régimen de HD se considera muy estricto lo que implica que las personas tengan serias dificultades para adaptarse y hacer frente a los factores estresantes agudos y crónicos. Estos factores de estrés se relacionan con las restricciones en la dieta, la ingestión de líquidos, la polifarmacia, así como los aspectos psicosociales, los cambios en la percepción de sí mismos y el miedo a la inminente muerte relacionada con el tratamiento.

Así mismo, coinciden con lo encontrado por Bennett et $\mathrm{a}^{26}$, en un estudio que buscó medir el bienestar subjetivo de las personas con enfermedad renal terminal en $\mathrm{HD}(n=172)$ respecto a un cohorte de población general, concluye que los pacientes en esta terapia dialítica reportan menor satisfacción con la vida en general, con los logros alcanzados, la relación y la seguridad personal en comparación con la población general. Señalan los autores que el bienestar subjetivo es un indicador importante de la calidad de vida de estas personas que debe ser considerado por los clínicos. Sin duda, estos componentes se constituyen en indicadores claves de intervención en la práctica y en fenómenos de interés investigativo sobre los que se debe ahondar.

Ahora bien los componentes de conocimiento (nociones e ideas respecto a la enfermedad y las terapéuticas farmacológicas y no farmacológicas), instrumental (habilidades y destrezas, así como las competencias para realizar las labores de cuidado, que incluyen la administración de medicamentos, alimentación, deambulación, baño y vestido) y anticipación (planeación ante la ocurrencia de un evento inesperado, la preparación para un examen y la identificación de riesgos) reportan una tendencia hacia el nivel medio, siendo llamativo este hallazgo teniendo en cuenta que los procesos de seguimiento y educación que brinda el equipo de salud generalmente se enfocan en estos aspectos, sobre los que se esperaría tener mayor impacto. Esto indica que los equipos deben analizar el abordaje actual del proceso educativo, con el fin de impactar en la dimensión anticipación.

Es de anotar que la educación en salud para enfermos renales en terapias dialíticas se constituye en un reto para el equipo interdisciplinario, estudios describen las bajas tasas de adherencia al régimen de alimentación ${ }^{27,28}$, siendo evidente la importancia de considerar modelos de cambio de comportamiento para facilitar la modificación de estilos de vida ${ }^{29}$ y en la exploración de otras variables como la fase de la cronicidad, los factores motivacionales, el refuerzo en la educación e incluso la percepción que tienen los usuarios de la terapia dialítica y el significado que le atribuyen a modificar 0 no las conductas propuestas.

Finalmente el componente de relación e Interacción Social (relaciones sociales así como las redes de apoyo primarias y secundarias que se tienen y hacen parte del diario vivir) reporta los niveles más altos, constituyéndose en un factor amortiguador que puede favorecer el fortalecimiento de la labor de cuidado en los usuarios. En un estudio realizado en Korea ${ }^{30}$ se reconoce que la mediación social es indispensable para mejorar la calidad de vida de los pacientes con enfermedad renal terminal, siendo relevante para evitar especialmente la depresión, la ansiedad y la fatiga.

\section{Conclusiones}

El perfil de la persona con ERC en la IPS Dialyser Bogotá es muy similar a lo que reporta la literatura frente a tipo de enfermedad y rango de edad. Se evidencia una prevalencia importante en el género masculino del grupo estudiado, así como en usuarios con bajos niveles de escolaridad, con ocupación hogar y de estrato socioeconómico bajo.

Los apoyos psicológico, familiar, religioso, económico y social y el nivel de satisfacción con esos apoyos con los que cuentan las personas con enfermedad renal son heterogéneos.

La competencia para el cuidado en el hogar (CUIDAR) de los pacientes con ERC en hemodiálisis es diversa, y dista de manera importante de ser adecuado para asu- 
mir el rol de cuidado garantizando calidad y seguridad en el mismo.

Con base en los resultados del estudio, se espera consolidar un diagnóstico nacional de la competencia para el cuidado en el hogar (CUIDAR) que incluya a los cuidadores familiares y otros centros de referencia de la institución. Es necesario, explorar otras variables que puedan incidir en la competencia, a través de ejercicios cualitativos que describan la experiencia y el significado que los usuarios atribuyen a este proceso.

Recibido: 12 febrero 16

Revisado: 8 marzo 16

Modificado: 20 abril 16

Aceptado: 27 abril 16

\section{Bibliografía}

1. Ministerio de Salud. Situación de la Enfermedad Renal Crónica en Colombia 2009. Disponible en: http://www.minsalud.gov.co/sites/rid/Lists/BibliotecaDigital/RIDE/INEC/CAC/Situacion $\% 20$ de\%20la\%20Enfermedad\%20Renal\%20Cronica\%20en\%20Colombia\%202009.pdf Consultado el 2 de febrero de 2015.

2. Situación de la Enfermedad Renal en Colombia, 2014, http://www.cuentadealtocosto.org/ byblos/Docs/SITUACION\%20ECR\%20COLOMBIA\%202014.swf.

3. Ministerio de Salud y Protección Social. Fondo Colombiano de Enfermedades de Alto Costo. Situación de la Enfermedad Renal Crónica en Colombia. 2013 Capitulo 1. Pp 9. En: http://www. cuentadealtocosto.org/byblos/Docs/Libro\%20 ERC\%202012.pdf Consultado el 9 de junio de 2015.

4. De Francisco AL, Sanjuan F, Foraster A, Fabado $S$, Carretero D, et al. Estudio epidemiológico de pacientes ancianos con insuficiencia renal crónica en hemodiálisis. Nefrologia 2008; 28 (1): 48-55.

5. Junqué $A$, Esteve $V$, Tomás $E$, López 0 , Iza G, Luceño $S$, et al. Resultado de un programa adaptado de ejercicio físico en pacientes ancianos en hemodiálisis. Enferm Nefrol 2015: Enero-Marzo; 18 (1): 11-18.

6. Quispe A, Quispe G, Quispe W. Complicaciones en pacientes con insuficiencia renal crónica terminal sometidos a hemodialisis regular en el Hospital Nacional Sur este Essalud Cusco, marzo-mayo 2002. Situa, 204; 13(1): Disponible en: http:// sisbib.unmsm.edu.pe/bvrevistas/situa/2004_nl/ Pdf/a07.pdf. Consultado el 10 de julio 2015.

7. Díaz P, García S, Arribas P. Valoración de la adherencia terapéutica de los pacientes de una unidad de hemodiálisis hospitalaria Enferm Nefrol 2013; 16 Suppl (1): 40/121.

8. Barrera L, Carrillo G, Chaparro L, Sánchez B. Modelo para abordar el cuidado de la enfermedad crónica en Colombia. Rev Orinoquía. 2015; 19(1): 85-95.

9. República de Colombia, Ministerio de Salud. Resolución 8430 de 1993. Por la cual se establecen las normas científicas, técnicas y administrativas para la investigación en salud. Ministerio de Salud de Colombia [Internet]. Octubre 4 de 1993. [citado 2015 marzo 23]. Disponible en: http:// goo.gl/rt5xgm.

10. Universidad Nacional de Colombia, Consejo Superior Universitario. Acuerdo 016 de 2011 por el cual se establece la Política Ambiental de la Universidad Nacional de Colombia [Internet]. Bogotá 18 de octubre de 2011. [citado 2015 marzo 23]. Disponible en: http://goo.gl/3XVkWv.

11. Chaparro L, Sánchez B, Carrillo G. Encuesta de caracterización del cuidado de la diada cuidador familiar-persona con enfermedad crónica. Rev. Ciencia y Cuidado. 2014; 11 (2): 31-45.

12. Carrillo G, Sánchez B, Vargas E. Desarrollo y pruebas psicométricas del instrumento "cuidar" versión corta para medir la competencia de cuidado en el hogar. Rev. Univ Ind Santander Salud. 2016; 48(2):222-231.

13. Sanabria M, Muñoz J, Trillos C, Hernández G, Latorre C, Díaz CS, et al. Dialysis outcomes in Colombia (DOC) study: A comparison of patient 
survival on peritoneal dialysis vs hemodialysis in Colombia. Kidney Internat. 2008; april 73: 165172.

14. Coronado Y, Lombo J, Correa I, Quintero N. Características clínicas y demográficas de los pacientes incidentes en diálisis crónica y su relación con el ingreso programado a diálisis. Acta Médica Colombiana. 2013; (38)3:138-14.

15. World Health Organization. Global Action Plan for the Prevention and Control of Noncommunicable diseases 2013-2020. (Consultado el 10 de julio 2015). En: http://www.who.int/nmh/events/ ncd_action_plan/en/.

16. Lucumí D, Gómez L. Accesibilidad a los servicios de salud en la práctica de citología reciente de cuello uterino en una zona urbana de Colombia. Rev Esp Salud Pública. 2004; 78(3):367-377.

17. Lewis MJ. Análisis de la situación del cáncer cervicouterino en América Latina y el Caribe. Washington, D.C.: OPS; 2004 [consultado el 29 de junio de 2014] En: http://www.paho.org/ Spanish/ AD/DPC/NC/pcc-cc-sit-lac.pdf.

18. Barreto S, Figueiredo R. Doença crônica, auto-avaliação de saúde e comportamento de risco: diferença de gênero. Rev. Saúde Pública . 2009 Nov; 43(Suppl 2): 38-47.

19. Barrera I, Pinto N, Sánchez B. Situación del Cuidador Familiar. Caracterización de los cuidadores en América Latina. Bogotá. En Cuidando a los cuidadores familiares de personas con enfermedad crónica. Universidad Nacional de Colombia: Bogotá. 2010.

20. Shu-Chuan Y. Living alone, social support, and feeling lonely among the elderly. Social Behavior and Personality. 2004; 32(2): 129-138.

21. Leung K, Chen C, Lue B, Hsu S. Social support and family functioning on psychological symptoms in elderly Chinese. Archives of Gerontology and Geriatrics. 2007; 44 (2): 203-213.

22. Ballew S, Hannum S, Gaines J, Marx K, Parrish $J$. The role of spiritual experiences and activities in the relationship between chronic illness and psychological well-being. J Relig Health. 2012; 51 (4):1386-1396.
23. Lamers S, Bolier L, Westerhof G, Smit F, Bohlmeijer $\mathrm{E}$. The impact of emotional well-being on long-term recovery and survival in physical illness: a meta-analysis. J Behav Med. 2012; 35 (5):538-547.

24. Carrillo G, Barreto R, Arboleda L, Gutiérrez A, et al. Competencia para cuidar en el hogar de personas con enfermedad crónica y sus cuidadores en Colombia. Rev. Fac Medicina Universidad Nacional de Colombia. 2015. En prensa.

25. Quinan P. Control and coping for individuals with end stage renal disease on hemodyalisis: a postion paper. CANNT J. 2007; 17(3):77-84.

26. Bennett $P$, Weinberg $M K$, Bridgman $T$, Cummins RA. The happiness and subjective well-being of people on haemodialysis. J Ren Care. 2015; 41 (3):156-161.

27. Durose $C L$, Holdsworth $M_{\text {, }}$ Watson $V$, Przygrodzka F. Knowledge of dietary restrictions and the medical consequences of noncompliance by patients on hemodialysis are not predictive of dietary compliance. J Am Diet Assoc 2004; 104 (1):35-41.

28. Karavetian M, de Vries $N$, Elzein $H$, Rizk R, Bechwaty $F$. Effect of behavioral stage-based nutrition education on management of osteodystrophy among hemodialysis patients, Lebanon. Patient Educ Couns. 2015; 98(9): 1116-22.

29. Spahn J, Reeves R, Keim K, Laquatra I, Kellogg $M$, Jortberg $B$, et al. State of the evidence regarding behavior change theories and strategies in nutrition counseling to facilitate health and food behavior change. J Am Diet Assoc 2010;110 (6):879-91.

30. Lee JE, Kim K, Kim JS. Factors Influencing Quality of Life in Adult Hemodialysis Patients With End-Stage Renal Disease. J Nurs Res. 2015; 23(3): 181-8. 